\title{
The Simplest Explanation is Usually the Correct One (A Medical Case in Which the "Ockham's Razor" was Fulfilled)
}

\author{
Di Pizio Lorenzo ${ }^{1}$, Schiavone Cosima ${ }^{2}$, Grappasonni Giulia ${ }^{2}$, Di Pizio Valerio ${ }^{2}$, Cipollone Francesco ${ }^{2}$ \\ and Guagnano Maria Teresa* \\ ${ }^{1}$ Internal Medicine, Department of Medicine and Science of Aging, University of Chieti, Italy \\ ${ }^{2}$ Resident of Internal Medicine, Department of Medicine and Science of Aging, University of Chieti, Italy
}

*Corresponding author: Guagnano Maria Teresa, Associate Professor of Internal Medicine, Department of Medicine and Science of Aging, University of Chieti, Italy

\section{ARTICLE INFO}

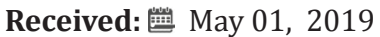

Published: 蔧 May 20, 2019

\section{ABSTRACT}

Citation: Di Pizio Lorenzo, Schiavone C, Grappasonni Giulia, Di Pizio Valerio, Cipollone F, Guagnano Maria T. The Simplest Explanation is Usually the Correct One (A Medical Case in Which the "Ockham's Razor" was Fulfilled). Biomed J Sci \& Tech Res 18(2)-2019. BJSTR. MS.ID.003113.

\section{Case Report}

A 77-year-old female patient was admitted at the Internal Medicine Unit for symptoms of fever lasting for about a week despite of an antibiotic treatment with levofloxacin (maximum body temperature of about $38^{\circ} \mathrm{C}$ ), asthenia, night sweats and itching in the upper and lower limbs and in the abdomen. She was affected by chronic ischemic heart disease, arterial hypertension, carotid and lower limbs of arteriopathy, chronic vascular leukoencephalopathy and mild depression. She was treated with an angiotensin converting enzyme (ACE) inhibitor, beta-blocker (carvedilol), acetylsalicylic acid, atorvastatin, torsemide and lansoprazole. On first physical examination, the patient showed mildly rough respiratory sounds, a moderate (2/6) aortic systolic murmur and enlarged lymph nodes of both later cervical sides. She had a temperature of about $37.7^{\circ} \mathrm{C}$. Table 1: Laboratory values at admission.

\begin{tabular}{|c|c|c|c|c|c|}
\hline Parameters & Value & Normal Range & Parameters & Value & Normal range \\
\hline Haemoglobin (g/dl) & 10.1 & $13-16$ & Albumin (g/dl) & 2.7 & $3.5-5.2$ \\
\hline Mean corpuscular volume (fl) & 83 & $77-95$ & $\alpha 1$-globulins (\%) & 7.5 & $2.9-4.9$ \\
\hline Mean cell haemoglobin (pg) & 25.7 & $25-35$ & $\alpha 2$-globulins (\%) & 15 & $7.1-11.8$ \\
\hline White blood cells $(* 103 / \mu \mathrm{l})$ & 7.9 & $4-10$ & $\gamma$-globulins (\%) & 14.1 & $11,1-18,8$ \\
\hline Neutrophils $(* 103 / \mu \mathrm{l})$ & 5.69 & $2.1-7.1$ & Iron blood $(\mu \mathrm{g} / \mathrm{dl})$ & 27 & $37-170$ \\
\hline Lymphocytes $(* 103 / \mu l)$ & 1.06 & $1.1-3$ & Ferritin (ng/ml) & 268.7 & $8-252$ \\
\hline Sodium (mmol/l) & 134 & $136-146$ & Transferrin (mg/dl) & 147 & $200-360$ \\
\hline Potassium (mmol/l) & 4 & $3.5-5.1$ & Ca125 (IU/ml) & 82.7 & $0-37$ \\
\hline
\end{tabular}

Blood tests showed a moderate anemia, with hemoglobin values ranging around $9-10 \mathrm{~g} / \mathrm{dl}$, a mild progressive decrease in lymphocytes (from 1060/microliters to 720/microliters), an increase in inflammatory markers (erythrosedimentation rate and C-reactive protein). Protein electrophoresis showed an increase of acute phase proteins ( $\alpha 1$ - and $\alpha 2$-globulins) despite of a decrease in albumin and total protein values. Blood iron and transferrin were low, ferritin was $268.7 \mathrm{ng} / \mathrm{ml}$ (mildly elevated). There was a specific mild increase in the oncological marker Ca 125. Blood and urine cultures, procalcitonin, and quantiFERON ${ }^{\circledR}$-TB Gold were negative (Table 1). The chest x-ray showed only an enlarged heart shape, which was consistent with an effect of her arterial hypertension. The electrocardiogram (ECG) was normal. 


\begin{tabular}{|c|c|c|c|c|c|}
\hline Calcium $(\mathrm{mmol} / \mathrm{l})$ & 2.3 & $2.1-2.55$ & Erythrosedimentation rate $(\mathrm{mm} / \mathrm{h})$ & 19 & $2-15$ \\
\hline Creatinine $(\mathrm{mg} / \mathrm{dl})$ & 0.7 & $0.52-1.04$ & C-reactive protein $(\mathrm{mg} / \mathrm{dl})$ & 5.59 & $0-0.5$ \\
\hline Aspartate transaminase $(\mathrm{IU} / \mathrm{l})$ & 19 & $14-36$ & Procalcitonin $(\mathrm{IU} / \mathrm{ml})$ & 0.19 & $0-0.5$ \\
\hline Alanine transaminase $(\mathrm{IU} / \mathrm{l})$ & 22 & $9-52$ & Blood cultures & Neg. & Neg. \\
\hline Total bilirubin $(\mathrm{mg} / \mathrm{dl})$ & 0,6 & $0.2-1.3$ & Urine cultures & Neg. & Neg. \\
\hline Total proteins $(\mathrm{g} / \mathrm{dl})$ & 5.2 & $6.3-8.2$ & QuantiFERON@-TB Gold & Neg. & Neg. \\
\hline
\end{tabular}

In suspicion of a lymphoproliferative disease, the patient performed an abdominal ultrasound, which showed an increased size of spleen (area of about 64 square centimeters, $\mathrm{cm}^{2}$ ), with markedly and widely inhomogeneous parenchymal and hilar echotexture, due to the presence of multiple irregular areas with poorly defined, hypoechoic margins. At the color doppler exam, these resulted mildly vascularized. Some lymph nodes of periaortic, pericaval and peripancreatic regions were increased in size (the largest had maximum dimensions of $2 \mathrm{~cm}$ and was localized in the pericaval site), had oval morphology, and visible echogenic hilum. Other lymph nodes of increased size were localized in the pelvic region. Because of such spread of the disease in the abdomen, in line with clinical features, we strongly suspected a Non-Hodgkin's lymphoma (Figure 1).
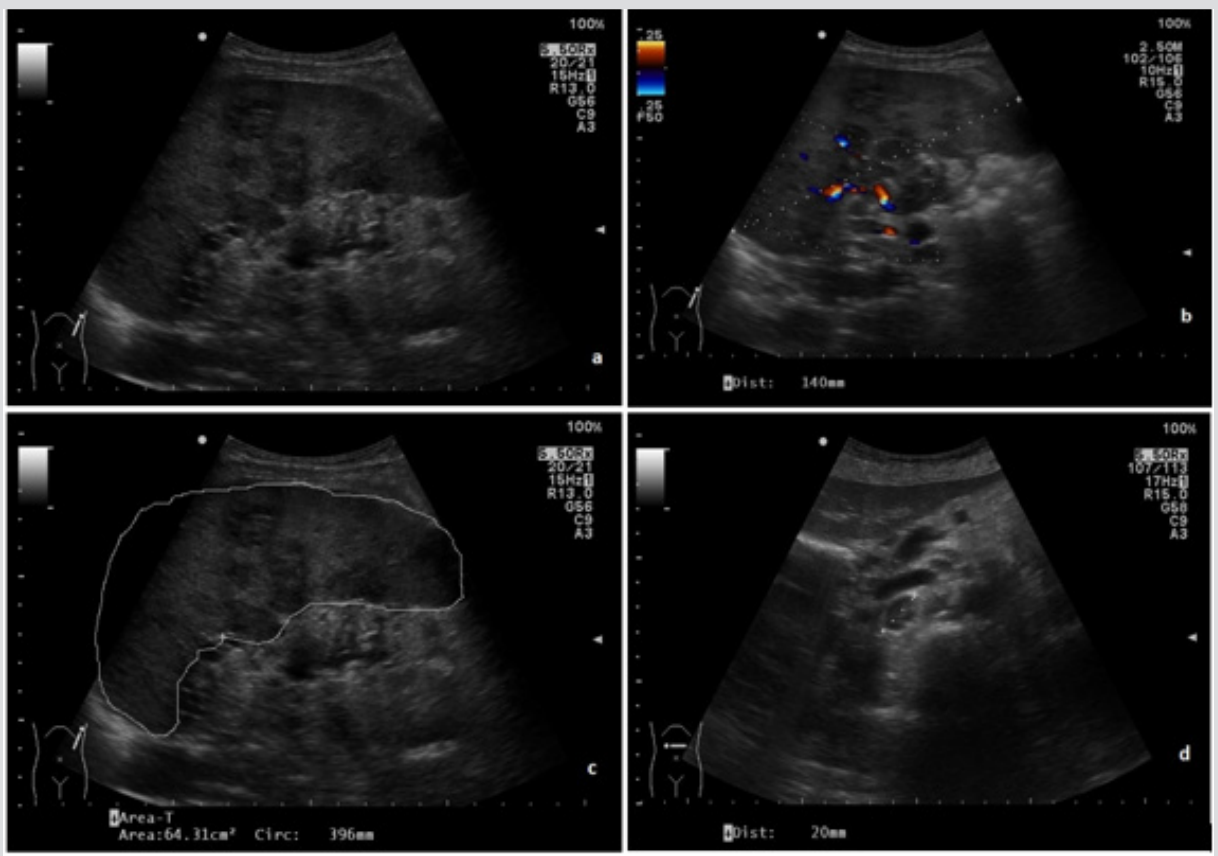

Figure 1: Upper abdominal ultrasound(B-mode).

a. Inhomogeneous spleen parenchyma with hypoechoic heterogeneous focal lesions

b. vascularisation and increased diameter of the spleen;

c. Increased area of the spleen;

d. Pericaval enlarged lymph node, with inhomogeneous echotexture and unrecognizable hilum.

The patient performed a contrast-enhanced thoracic and abdominal computed tomography (CT), which showed enlarged lymph nodes (short axis of maximum $12 \mathrm{~mm}$ ) at the left base of the neck, in both supraclavicular and retropectural regions, in celiac, paraortic, retrocaval and interaorto-caval areas. Enlarged lymph-nodes were also localized in the paratracheal site, in aortopulmonary window, in subcarinal and hilar area, bilaterally along the external iliac and common femoral vessels. In the left groin, some of them infiltrated ipsilateral internal obturator and adductor muscles, which appeared thickened and in homogeneously contrast enhanced. The spleen had a bipolar diameter of about
13 centimeters $(\mathrm{cm})$, an inhomogeneous parenchymal structure due to the presence of newly formed solid tissue extending to the hilum close to the tail of the pancreas. Some enlarged lymph nodes were along the splenic vessels, which appeared narrowed and poorly contrast enhanced as per compression/infiltration. The liver appeared slightly enlarged but free from focal lesions of pathological significance and the bile ducts were not dilated. Mild effusion layers were in both pleural spaces, in the perihepatic space and in Douglas' pouch. An osteoaddensing alteration of about $1 \mathrm{~cm}$ was in the soma of the first thoracic vertebra (D1) (Figure 2). 


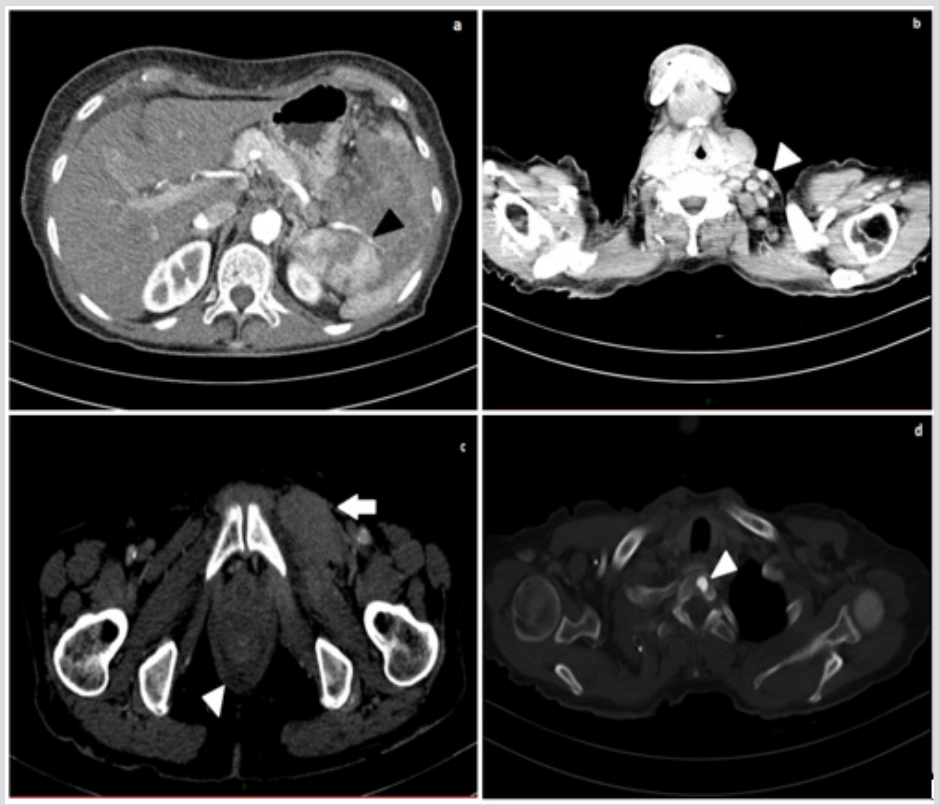

Figure 2:

a. Contrast-enhanced abdominal CT (arterial phase), showing inhomogeneous spleen parenchyma and narrowing of splenic vessels (black arrowhead);

b. Contrast-enhanced neck CT, showing enlarged later cervical lymph nodes (white arrowhead);

c. Contrast-enhanced CT of the pelvis: thickening of the left obturator muscle (white arrow) and mild effusion in the Douglas pouch (white arrowhead);

d. Contrast-enhanced CT: osteoaddensing lesion in the soma of the first thoracic vertebra D1 (white arrowhead).

The patient then performed a biopsy of left later cervical lymph nodes. The histological examination was consistent with a mixed cellularity subtype of classical Hodgkin lymphoma. The immunophenotyping was positive for CD30, CD15, PAX5, Fascine and negative for CD20 and CD3. In association with CT aspects, these clinical features were consistent with a stage IV disease, according to the 2014 Lugano classification (Cotswold's modifications applied to Ann Arbor classification) (Table 2). During the hospital stay, the patient was treated with supportive and antibiotic therapy (since the initial suspicion of infectious disease). She was then referred to a Hematologic Center to start a specific diagnostic and therapeutic iter.

Table 2: Lugano classification for staging of lymphomas (derived from Ann Arbor staging with Cotswolds modifications).

\begin{tabular}{|c|c|c|}
\hline \multicolumn{3}{|c|}{ Revised Staging System for Primary Nodal Lymphomas } \\
\hline Stage & Involvement & Extranodal (E) Status \\
\hline \multicolumn{3}{|c|}{ Limited } \\
\hline I & One node or a group of adjacent nodes & Single extra nodal lesions without nodal involvement \\
\hline II & Two or more nodal groups on the same side of the diaphragm & $\begin{array}{c}\text { Stage I or II by nodal extent with limited contiguous } \\
\text { extra nodal involvement }\end{array}$ \\
\hline II bulky, & II as above with "bulky" disease & Not applicable \\
\hline \multicolumn{3}{|c|}{ Advanced } \\
\hline III & $\begin{array}{l}\text { Nodes on both sides of the diaphragm; nodes above the } \\
\text { diaphragm with spleen involvement }\end{array}$ & Not applicable \\
\hline IV & Additional noncontiguous extralymphatic involvement & Not applicable \\
\hline
\end{tabular}

Note: Extent of disease is determined by positron emission tomography-computed tomography for avid lymphomas and computed tomography for nonavid histologies. Tonsils, Waldeyer's ring, and spleen are considered nodal tissue.

\section{Discussion}

Hodgkin lymphoma (HL) is a lymphoproliferative disease characterized by the presence of neoplastic cells (Reed-Sternberg cells) in a variable inflammatory milieu consisting of cells (eosinophils, neutrophils, macrophages, lymphocytes, plasma cells, fibroblasts) and different degrees of fibrosis. It arises from germinal center or post-germinal center B cells. Based on the histopathologic and immunophenotypic appearances, it can be divided in two major 
subgroups, nodular lymphocyte predominant Hodgkin Lymphoma (NLPHL) and classic Hodgkin lymphoma (CHL). The latter is further divided in four subtypes, based on differences in the appearance of the tumor cells and the composition of the reactive background: nodular sclerosis, mixed cellularity, lymphocyte rich, lymphocyte depleted [1]. Hodgkin and Non-Hodgkin lymphomas (NHL) represent about $5 \%$ of malignant neoplasms. It is often confined to lymph nodes, but $40 \%$ may show extra nodal involvement, more frequently in spleen and liver. The involvement of abdominal lymph nodes is more frequent in Non-Hodgkin lymphoma (NHL) than in HL [2].

The extra nodal extension of the disease is essential to establish the type of treatment required [3]. The splenic involvement in HL and NHL has almost an overlapping prevalence and is often accompanied by liver involvement. Some Authors have observed that splenic involvement in Hodgkin's lymphoma is correlated with a younger age [4]. The splenic involvement is presumably via the hematogenous route [5]. Primary lymphomas of the spleen are very rare (less than $1 \%$ ) and usually are represented by NonHodgkin lymphomas [6]. Splenic ultrasound, in cases of lymphoma involvement, is characterized by a sensitivity of $54 \%$ and a specificity close to $100 \%$ [7]. The increase in size of the organ is neither a necessary nor sufficient condition to confirm (or exclude) the presence of the disease. More frequently, splenomegaly occurs if the involvement is widespread $[2,6]$.

At B-mode ultrasound examination, we can distinguish four main echo-texture patterns for splenic lymphomas: diffuse infiltration, "small-nodular" pattern, "large-nodular" pattern and "bulky" lesion [6-8]. Typically, Hodgkin lymphomas and low-grade Non-Hodgkin lymphomas show a diffuse infiltration or focal lesions with a size of less than $3 \mathrm{~cm}$. Conversely, high-grade Non-Hodgkin lymphomas usually appear as lesions greater than $3 \mathrm{~cm}$ in size [6]. The ultrasound features of splenic lesions correlate with the severity of the pathology, being a diffuse pattern consistent with a low-intermediate grade and nodular lesions with an intermediate-high grade [8]. The lesions frequently appear hypoechoic or anechoic (so-called "cystic-like"), but they can rarely be isoechoic, hyperechoic or isoechoic with a hypoechoic halo (more rarely with a "target" appearance), and sometimes they can coexist. Hyperechogenic lesions may actually correlate with reactive benign changes in the splenic parenchyma.

Splenic lymphomatous foci usually have a weak vascular signal, especially useful for differentiating benign cystic lesions from "cystic-like" nodules, which show an intralesional vascular signal. With regard to vascular indices, no significant changes in resistive index (RI) or average flow velocity in the portal vein were observed (parameters that would seem to be linked to the grade of congestion of the portal system). Instead, a significant increase in the flow volume in the portal vein was observed, probably linked to the "hyperplastic" lymphoma mechanism. This explanation seems to be valid also in the case of transplanted patients, in whom similar results have been observed [9]. For Hodgkin lymphoma staging and treatment planning, 18F-Fluorodeoxyglucose positron emission tomography/ computed tomography (18F-FDG PET/CT) represents the imaging exam of choice, but sometimes ultrasound may be superior to other imaging techniques in detecting splenic disease [10].

Contrast-enhanced ultrasonography (CEUS) with second generation contrast agents (Sonovue $\AA$ ) has facilitated the diagnosis of lymphoma splenic lesions, especially in detection and characterization of focal lesions (while it shows diagnostic limitations in case of widespread involvement of the organ). In particular, lesions with a diameter greater than 5 millimeters $(\mathrm{mm})$ show variable behavior in the arterial phase, while in the parenchymal phase they appear invariably hypoechoic in comparison with the surrounding parenchyma. Specifically, for Hodgkin lymphomas, Catalano et al. described increased perfusion of focal Hodgkin lymphoma on contrast-enhanced sonography during the arterial phase [8-11]. The increased perfusion remained visible throughout the later phases. However, the role of contrastenhanced sonography in the differential diagnosis of Hodgkin lymphoma has been discussed controversially and has very limited clinical relevance for the diagnosis of splenic lymphomas.

Two-dimensional real-time shear wave elastography (SWE) is an imaging technique that uses acoustic radiation force to stimulate the production of a linear vibration source and then uses special high-speed imaging to track the displacement of each point on the path of shear wave propagation [12]. Currently, the main application of shear-wave elastosonography (SWE) in the study of the spleen in hematological diseases is reserved for the study of the splenic response to primary myelofibrosis. There are still few studies on the use of SWE in splenic involvement in lymphoproliferative diseases $[13,14]$. About lymph nodes, the data from scientific literature are mainly related to later cervical and superficial ones. It is not possible to make a differential diagnosis of Hodgkin lymphoma and Non-Hodgkin lymphoma by ultrasound examination. They appear increased in size with a short axis equal to or greater than $10 \mathrm{~mm}$. They tend to be roundish, hypoechoic, with well-defined margins, and the hilum that loses its echogenicity. Many of these features are indistinguishable from those of metastatic lymph nodes. At the color doppler investigation, both the hilar and peripheral vessels are visualized, as opposed to metastatic lymph nodes, where the extensive necrosis and keratinization alter the hilar vascular tree6 [15].

The lymph node size and vascularization are good parameters for assessing the response to treatment. With regard to vascular indices, even if the literature is poor in this regard, it is generally agreed that there is an increase in RI (ranging from 0.64 to 0.84 ) compared to inflammatory or metastatic lymph nodes. It is generally believed that the RI of lymphomatous nodes is higher than those of reactive, tuberculous and normal nodes, and are lower than those of metastatic nodes [15]. US elastography appears to be a promising tool for diagnosing benign and malignant lymph 
nodes diseases, in selected populations [16]. In general, metastases are stiff, tuberculosis and lymphoma are less rough; benign disease has a soft consistency. In particular, it has been reported that rockhard adenopathy is associated with Hodgkin disease theoretically and clinically, whereas other scholars believe that the degree of stiffness is different. According to Tan and al., lymphoma is not remarkably hard but a little stiff when it is invasive and different from reactive hyperplasia, which is soft.

Furthermore, in their study, 4 cases of lymphoma showed increased elasticity, which may have been associated with an increase in the tension caused by excessive proliferation of tumor tissue, and fibrosis appeared in internal lesions. Because of fewer cases and different pathologic types, the stiffness features of lymphoma need to be studied further. SWE is unlikely to be suitable in clinical practice for screening of nodes for malignancy. Conversely, the high specificity may be useful for selecting a subgroup of nodes at very high risk of being malignant and thus could undergo FNAC irrespective of gray-scale findings [17]. Contrast-enhancement ultrasonography (CEUS) demonstrates more lymph node vessels, which allows more accurate characterization of nodal vascularity. CEUS is useful to visualize a heterogeneous and irregular pattern of enhancement. Currently, the European Federation of Societies for Ultrasound in Medicine and Biology (EFSUMB) does not recommend the exam for the study of lymphadenopathy [18].

A useful application is dynamic CEUS, a study of changes in quantitative parameters during ultrasound contrast agent administration (second generation, Sonovue $₫$ ) The time-intensity curve (TIC) analysis has been applied to evaluate changes in wash-in phase, time to peak, intensity and wash-out phase of the ultrasound contrast agent, in patients receiving chemotherapy. In general, diseased lymph nodes have a more rapid wash-in, and wash-out and a lower time to peak time [19]. Dynamic sonographic contrast enhancement in Hodgkins and non-Hodgkins lymphoma showed a delay in the time to peak enhancement after treatment. Even the values of enhancement intensity and peak intensity appear to be lower after treatment. Dynamic contrast scanning using ultrasound is advantageous over similar techniques using CT or MRI in that it is radiation-free, has a high spatial resolution yet maintains a high frame rate, and can be performed repeatedly during the same examination. It appears to provide a new, timedependent dimension in the assessment of lymph node pathology and supplements the morphological information provided by grey scale and Doppler sonographic interrogation[15].

\section{Conclusion}

In modern medicine, ultrasound examinations definitely are handy tools to draw pathways of diagnostic workups. In our patient, given the clinical presentation, our first suspicion was about a lymphoproliferative disease. Ultrasound was useful to strengthen our hypothesis. Definitive diagnosis and staging were easily made with histology, immunophenotyping and contract-enhanced CT scan. That's why, in this specific case, we can affirm that "simpler solutions are more likely to be correct than complex ones", as the philosopher William of Ockham stated in the $14^{\text {th }}$ century.

\section{References}

1. Castoldi G, Liso V (2013) Malattie del sangue e degli organi ematopoietici (6 $6^{\text {th }}$ ed) McGraw-Hill (Ed).

2. Manzella A, Borba Filho P, DIppolito G, Farias M (2013) Abdominal Manifestations of Lymphoma: Spectrum of Imaging Features. ISRN Radiol 483069.

3. Iannitto E, Tripodo C (2011) How I diagnose and treat splenic lymphomas. Blood 117(9): 2585-2595.

4. Di Stasi M, Cavanna L, Fornari F, Vallisa D, Buscarini E, et al. (1995) Splenic lesions in Hodgkin's and non-Hodgkin's lymphomas. An ultrasonographic study. Eur J Ultrasound 2(2): 117-124.

5. Silinuoto T, Päivansalo M, Alavaikko M (1991) Ultrasonography of spleen and liver in staging Hodgkin's disease. Eur J Radiol 13(3): 181-186.

6. Schiavone C, Romano M (2013) Manuale Italiano di Ecografia Internistica. ( $2^{\text {nd }}$ edn.). Antonio Delfino (Ed).

7. Görg C, Faoro C, Tillmann B, Tebbe J, Neesse A, et al. (2011) Contrast enhanced ultrasound of splenic lymphoma involvement. Eur J Radiol 80(2): 169-174.

8. Benter T, Klühs L, Teichgräber U (2011) Sonography of the spleen. J Ultrasound Med 30(9): 1281-1293.

9. Piscaglia F, Donati G, Cecilioni L, Celli N, Stagni B, et al. (2002) Influence of the Spleen on Portal Haemodynamics: A Non-invasive Study with Doppler Ultrasound in Chronic Liver Disease and haematological Disorders. Scand J Gastroenterol 37(10): 1220-1227.

10. Cheson BD, Fisher RI, Barrington SF, Cavalli F, Schwartz LH, et al. (2014) Recommendations for initial evaluation, staging, and response assessment of Hodgkin and non-Hodgkin lymphoma: the Lugano classification. J Clin Oncol 32(27): 3059-3068.

11. Catalano O, Lobianco R, Sandomenico F, DElia G, Siani A (2003) Real-time contrast-enhanced ultrasound of the spleen: examination technique and preliminary clinical experience. Radiol Med 106(4): 338-356.

12. Tan S, Miao LY, Cui LG, Sun PF, Qian LX (2017) Value of Shear Wave Elastography Versus Contrast Enhanced Sonography for Differentiating Benign and Malignant Superficial Lymphadenopathy Unexplained by Conventional Sonography. J Ultrasound Med 36(1): 189-199.

13. Mazur R, Celmer M, Silicki J, Hołownia D, Pozowski P, et al. (2018) Clinical applications of spleen ultrasound elastography - a review. J Ultrason 18(72): 37-41.

14. Webb M, Shibolet O, Halpern Z, Nagar M, Amariglio N, et al. (2015) Assessment of Liver and Spleen Stiffness in Patients with Myelofibrosis Using FibroScan and Shear Wave Elastography. Ultrasound 31(3): 166169.

15. Ahuja AT, Ying M, Ho SY, Antonio G, Lee YP, et al. (2008) Ultrasound of malignant cervical lymph nodes. Cancer Imaging 8: 48-56.

16. Choi YJ, Lee JH, Baek JH (2015) Ultrasound elastography for evaluation of cervical lymph nodes. Ultrasonography 34(3): 157-164.

17. Bhatia KS, Cho CC, Tong CS, Yuen EH, Ahuja AT (2012) Shear wave elasticity imaging of cervical lymph nodes. Ultrasound Med Biol 38(2): 195-201.

18. Sidhu PS, Cantisani V, Dietrich CF, Gilja OH, Saftoiu A, et al. (2018) The EFSUMB Guidelines and Recommendations for the Clinical Practice of Contrast-Enhanced Ultrasound (CEUS) in Non-Hepatic Applications: Update 2017 (Long Version). Ultraschall Med 39(2): e2-e44.

19. Xin L, Yan Z, Zhang X, Zang Y, Ding Z, et al. (2017) Parameters for Contrast-Enhanced Ultrasound (CEUS) of Enlarged Superficial Lymph Nodes for the Evaluation of Therapeutic Response in Lymphoma: A 
Preliminary Study. Med Sci Monit 23: 5430-5438.

\section{ISSN: 2574-1241}

DOI: 10.26717/BJSTR.2019.18.003113

Schiavone Cosima. Biomed J Sci \& Tech Res

(c) (i) This work is licensed under Creative

Submission Link: https://biomedres.us/submit-manuscript.php

$\begin{array}{ll}\text { BIOMEDICAL } & \text { Assets of Publishing with us } \\ \text { RESEARCHES } & \text { - Global archiving of articles } \\ & \text { - Immediate, unrestricted online access } \\ & \text { - Rigorous Peer Review Process } \\ \end{array}$

